\title{
Necrotizing sarcoid granulomatosis: A case report and review of progresses in this disease
}

\author{
Guido Epping ${ }^{1}$, Carlos A. Schwengle ${ }^{1}$, Riena P. Aliredjo ${ }^{2}$, Michiel Wagenaar ${ }^{1}$ \\ ${ }^{1}$ Department of Pulmonary Medicine, Medisch Spectrum Twente, Enschede, The Netherlands; g.epping@mst.nl \\ ${ }^{2}$ Department of Pathology, Medisch Spectrum Twente, Enschede, The Netherlands.
}

Received 9 May 2011; revised 27 June 2011; accepted 18 July 2011.

\begin{abstract}
We present the case of a young male who visited the emergency room with progressive dyspnea and left sided chest pain. A chest X-ray was performed which showed a pattern of diffuse nodular and reticular opacities. The diagnosis of Necrotizing Sarcoid Granulomatosis (NSG) was made by histopathological examination of a lung biopsy specimen and by excluding other causes of granulomatous disease. He was treated with corticosteroids. Because of persistent extrapulmonary (ocular) involvement a maintenance dose of prednisone was needed. Necrotizing Sarcoid Granulomatosis is a granulomatous disease with necrosis and vasculitis of unknown cause. The resemblance between NSG and (nodular) sarcoidosis is strong, but subtle differences exist. The prognosis is good and therapy is usually not necessary. However in some cases there is a need for therapy with corticosteroids, depending on the involved organs and/or the degree of impairment.
\end{abstract}

Keywords: Necrotizing Sarcoid Granulomatosis; Sarcoidosis; Extrapulmonary Involvement; Computed Tomography; Pathology

\section{CASE PRESENTATION}

A 21 year old non-smoking male was presented at the emergency department of our hospital. He suffered from pain in his right shoulder and on the left side of his chest, with progressive shortness of breath for two days. He had a productive cough with clear blank sputum. There was no haemoptysis, nor were there constitutional symptoms like fever or night sweats. There were no complaints that could be related to a vasculitis-like disease, e.g. vision problems, joint aches, skin problems or abdominal discomfort. He had a blank history, and didn’t use any medication.
Our patient was tachypnoeic with an oxygen saturation of $98 \%$ with 3 litres of supplemental oxygen per minute. His blood pressure was $110 / 70 \mathrm{mmHg}$ with a regular pulse of 115/minute. He had a low grade fever, with a temperature of $38,2^{\circ} \mathrm{C}$. There were no palpable lymph nodes. Chest examination revealed wheezing on both sides and crackles at the bases. There were no other abnormalities at the physical examination.

The posteroanterior view of the chest X-ray showed diffuse reticular and nodular opacities of both lungs (Figure 1). A computed tomography of the chest and upper abdomen (Figure 2) showed mediastinal and right hilar lymphadenopathy. Diffusely dispersed alveolar opacities were present in both lungs, which were more pronounced in the lower lobes.

Laboratory findings showed an elevated erythrocyte sedimentation rate. The C-reactive protein and white cell counts were both minimally elevated. The serum angiotensin converting enzyme (ACE) level was low. Antinucleair antibodies (ANA) and anti-neutrophil cytoplasmatic antibodies (ANCA) were both negative. Analysis of his urine showed no abnormalities. Pulmonary function tests showed a restrictive pattern (TLC $=53 \%$ of reference) with a diminished diffusion capacity $\left(\mathrm{D}_{\mathrm{L}} \mathrm{CO}=\right.$ 48\%). Arterial blood gas analysis (ABGA) breathing room air showed a mild hypoxemia $\left(\mathrm{pO}_{2} 9.6 \mathrm{kPa}\right)$, with normocapnia $\left(\mathrm{pCO}_{2} 5.1 \mathrm{kPa}\right)$.

We performed extensive microbiological testing. All tests on tuberculosis were negative (Mantoux; Quantiferon TB Gold test; Acid-fast staining; PCR tests and cultures). Serological tests on HIV, viral respiratory infections (adenovirus; influenza A/B; parainfluenzae 1/2/3/; $R S$ virus) and so-called atypical pathogens (chlamydia pneumoniae and psittaci; coxiella burnetii; legionella pneumophila; mycoplasma pneumonia) were all negative.

Bronchoscopic examination was performed. No endobronchial abnormalities were noted. Central biopsy and broncho-alveolar lavage (BAL) showed no signs of malignancy or (specific) inflammation. Cultures were 


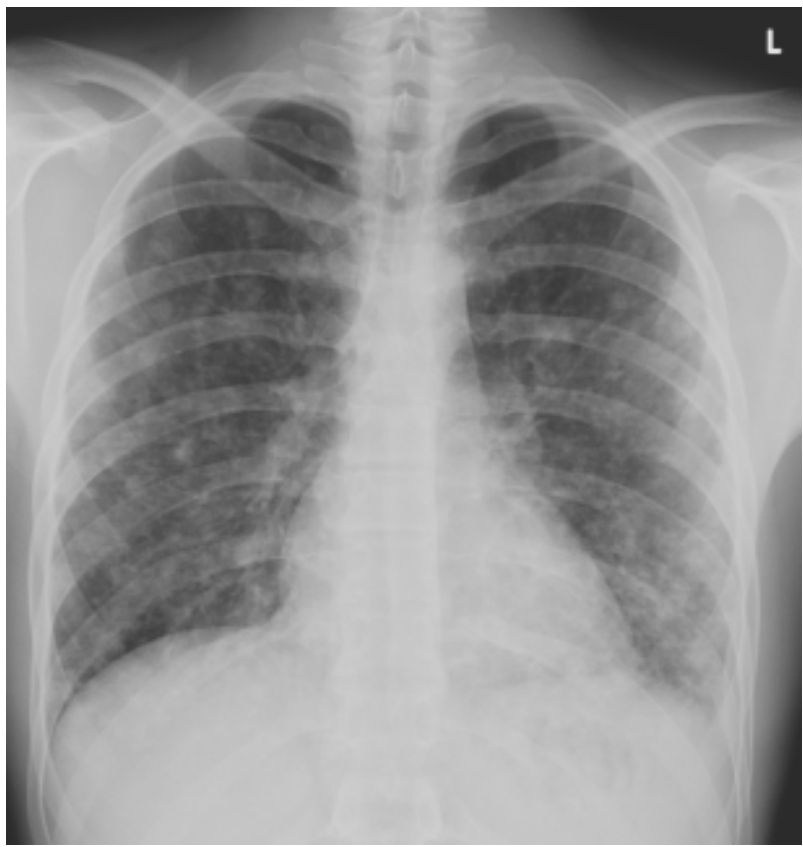

Figure 1. Chest X-ray (PA view) with diffusely dispersed reticular and nodular opacities in both lungs.

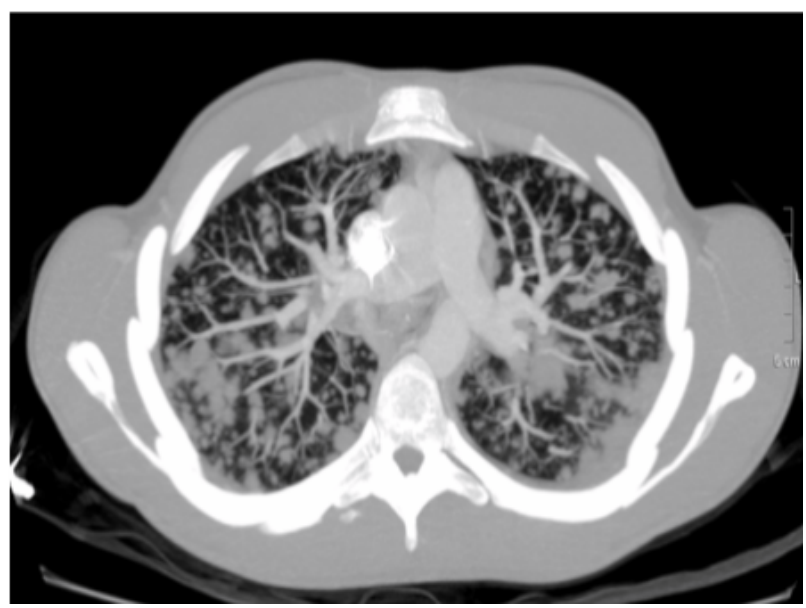

Figure 2. Computed tomography of the chest with enlarged lymph nodes both mediastinal and hilar, and diffusely dispersed nodular opacities.

negative. The BAL contained a lymphocytosis and neutrophilia, with in the differential: $1 \%$ eosinophils (normal $<1 \%-2 \%$ ), $9 \%$ neutrophils (normal < 3\%), 58\% macrophages (normal $>80 \%$ ) and 32\% lymphocytes (normal $<15 \%$ ). The CD4/CD8 ratio was slightly elevated $(3,0)$ and the CD103/CD4 ratio was decreased $(0,12)$. The BAL results with lymphocytosis, slightly elevated CD4/CD8 ratio and a diminished CD103/CD4 ratio were compatible with sarcoidosis.

Vasculitis and malignancy were less probable but could not be excluded. A video-assisted thoracoscopic (VATS) biopsy was performed of the right lung. Histo- logical examination showed nodular abnormalities consisting of granulomas with epitheloid cells, Langerhans type giant cells and necrosis. The granulomas were dispersed through the parenchyma and along the bronchi a reflection of vasculitis of the adjacent blood vessels was seen (Figure 3). The pathological and clinical diagnosis therefore was: Necrotizing Sarcoid Granulomatosis.

Because of the extensive pulmonary involvement and the seriousness of his complaints we decided to treat our patient with corticosteroids. We started with a dose of $60 \mathrm{mg}$ of prednisone a day and gradually tapered the dose till zero, over a period of six months. With this regime complete recovery was achieved, with normalization of his chest X-ray. His chest pain was completely disappeared. However, after seven months he developed a sicca like syndrome of the eye. He was examined by an ophthalmologist and an octreotide scan was performed, the diagnosis of dacryoadenitis was made. Prednisone was reintroduced in a dose of $30 \mathrm{mg}$ a day. Again the dose was gradually reduced. This time we were unable to reduce it below a dose of $10 \mathrm{mg}$ a day, because of a flare of his pulmonary complaints. With a maintenance dose of ten milligrams he is free of symptoms for a year now.

\section{CURRENT UNDERSTANDING OF NSG IN HUMAN HEALTH}

NSG is a rare entity which was first described by Liebow in 1973. [1-3] The incidence of NSG is not known. NSG is more common in females than males and most are non-smokers. [4] Few cases are published with a total of seven publications since the case-series by Churg and Gupta in 1979. [4] The age of presentation varies, with distribution of cases between the second and the sixth decade of life. [2,4].

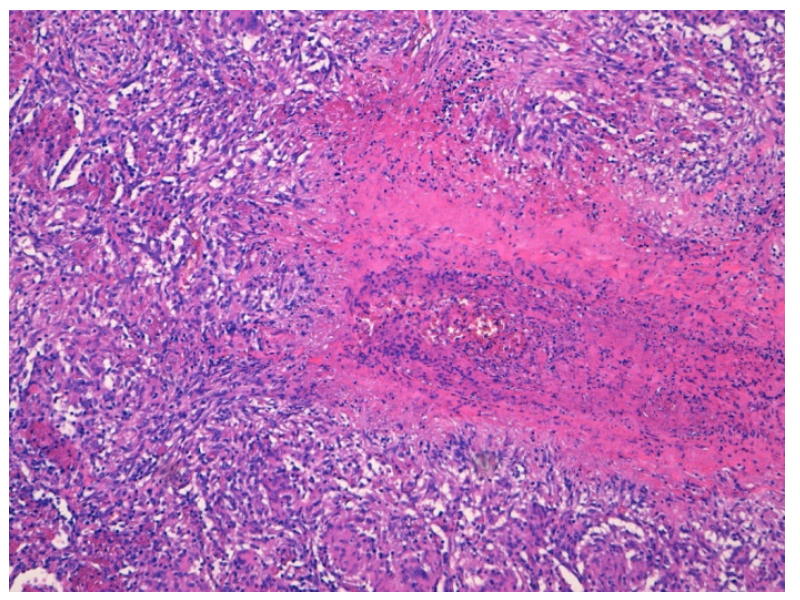

Figure 3. VATS biopsy specimen: in the center vasculitis surrounded by tissue with multiple granulomas (HematoxylinEosin stain, magnification X 50). 
NSG was originally characterized by the following three characteristics [2]: 1) on pathological examination there was a background of sarcoid-like granulomas, a prominent and usually granulomatous vasculitis, and varying degrees of necrosis, often superimposed on a mass of confluent granulomas; 2) on chest X-ray, there were pulmonary nodules but no enlarged hilar lymph nodes; and 3) a clinically benign course with minimal or no therapy.

There are histological resemblances between NSG and sarcoidosis but also with vasculitis. The question whether NSG is a vasculitis with sarcoid reaction (angiocentric granulomatosis) or a variant of sarcoidosis, still remains unanswered. [5] Some believe it is a variant of nodular sarcoidosis e.g. nodular sarcoidosis with necrosis. [3,6] Others suggested that some cases of NSG are actually inactive granulomatous infections. [7] The etiology and pathogenesis of NSG is still unknown. [4]

NSG usually involves the lungs, but as in our case extra-pulmonary involvement has been described. There are case reports of pleural, eye, skin, liver, spleen, kidney and neurological involvement. [4,8-11]

The diagnosis of NSG can be suspected based upon clinical findings, laboratory tests, lung function and radiographic findings (chest $\mathrm{x}$-ray and CT scan). Patients can present with systemic symptoms, such as fatigue, fever, night sweats and weight loss. Extra-pulmonary disease can be symptomatic. Besides, patients can have respiratory symptoms such as cough, dyspnoea and chest pain. Physical examination is usually non-specific or completely normal. Although crackles at lung examination, joint swelling and splenomegaly can sometimes be found. All these findings are nonspecific.

Pulmonary function tests are usually normal. [4] Laboratory findings are non-specific and non-diagnostic. The erythrocyte sedimentation rate is usually elevated. ACE is typically low and ANCA is usually negative. [4] Both sarcoidosis and vasculitis however, cannot be ruled out by these laboratory investigations. A chest X-ray or Computed Tomography of the chest can show alveolar opacities or infiltrates, a solitary nodule or diffuse nodules, hilar and/or mediastinal lymphadenopathy, pleural thickening and cavitations. There are no specific radiographic findings that can distinguish NSG from other granulomatous diseases with certainty. [4]

To confirm the diagnosis a histological examination of lung tissue or other involved organs is necessary. A representative lung biopsy can be obtained through a VATS or open lung procedure.

Prognosis is generally good with more than $80 \%$ complete recovery, either spontaneous or with therapy. $[2,12]$ There are no guidelines for the treatment of NSG. Depending upon disease progression and the degree of impairment, corticosteroids should be considered.

\section{CONCLUSIONS}

We present a patient diagnosed with Necrotizing Sarcoid Granulomatosis, with extrapulmonary involvement. We reviewed the available literature and addressed different aspects of this disease. NSG is viewed by some as a vasculitis with sarcomatoid reaction, while others look at it as a variant of sarcoidosis. Either way it is a disease of unknown etiology, with a good prognosis that sometimes requires treatment with corticosteroids.

\section{REFERENCES}

[1] Liebow, A.A. (1973) The J. burns amberson lecture. Pulmonary angiitis and granulomatosis. American Review of Respiratory Disease, 108, 1-18.

[2] Churg, A., Carrington, C.B. and Gupta, R. (1979) Necrotizing sarcoid granulomatosis. Chest, 76, 406-413. doi:10.1378/chest.76.4.406

[3] Churg, A. (1983) Pulmonary angiitis and granulomatosis revisited. Human Pathology, 14, 868-883. doi:10.1016/S0046-8177(83)80162-2

[4] Quaden, C., Tillie-Leblond, I., Delobbe, A., et al. (2005) Necrotising sarcoid granulomatosis: Clinical, functional, endoscopical and radiographical evaluations. European Respiratory Journal, 26, 778-785. doi:10.1183/09031936.05.00024205

[5] Rosen, Y. (2007) Pathology of sarcoidosis. Seminars in Respiratory and Critical Care Medicine, 28, 36-51. doi:10.1055/s-2007-970332

[6] Popper, H.H., Klemen, H., Colby, T.V. and Churg, A. (2003) Necrotizing sarcoid granulomatosis-Is it different from nodular sarcoidosis? Pneumologie, 57, 268-271. doi:10.1055/s-2003-39365

[7] Koss, M.N., Hochholzer, L., Feigin, D.S., Garancis, J.C. and Ward, P.A. (1980) Necrotizing sarcoid-like granulomatosis: clinical, pathologic, and immunopathologic findings. Human Pathology, 11, 510-519.

[8] Alberti, S., Falleni, M., Tarsia, P., et al. (2006) A 13-year-old female with shortness of breath and pleuritic chest pain. European Respiratory Journal, 28, 876-882. doi:10.1183/09031936.06.00021006

[9] Chittock, D.R., Joseph, M.G., Paterson, N.A.M. and McFadden, R.G. (1994) Necrotizing sarcoid granulomatosis with pleural involvement: Clinical and radiographic features. Chest, 106, 672-676. doi:10.1378/chest.106.3.672

[10] Dykhuizen, R.S., Smith, C.C., Kennedy, M.M., et al. (1997) Necrotizing sarcoid granulomatosis with extrapulmonary involvement. European Respiratory Journal, 10, 245-247. doi:10.1183/09031936.97.10010245

[11] Strickland-Marmol, L.B., Fessler, R.G. and Rojiani, A.M. (2000) Necrotizing sarcoid granulomatosis mimicking an intracranial neoplasm: Clinicopathologic features and review of the literature. Modern Pathology, 13, 909-913. doi:10.1038/modpathol.3880162

[12] Lynch III, J.P., Kazerooni, E.A. and Gay, S.E. (1997) Pulmonary sarcoidosis. Clinics In Chest Medicine, 18, 755-785. doi:10.1016/S0272-5231(05)70417-2 